\title{
MedBG: Lobbying oder Vertrauenspartner?
}

Die nationalrätliche Kommission für soziale Sicherheit und Gesundheit hat sich am 27. und 28. April mit dem neuen Medizinalberufegesetz befasst. Es geht darin nicht nur um die Ausbildung, sondern auch um die Bewilligung zur Berufsausübung. Dem Gesetzesvorschlag liegt ein relativ komplizierter Mechanismus zugrunde: Er sieht zunächst einmal die Schaffung einer Medizinalberufekommission mit Vertretern aus Bund, Kantonen, Universitäten und Berufsorganisationen vor - das Gesetz betrifft auch die Zahnärzte, die Apotheker und die Tierärzte. Es stellt den Kern von Verhandlungen unter Partnern mit den verschiedenartigsten Kompetenzen dar: So enthält es unter anderem die Aufsicht über die eidgenössischen Prüfungen wie wir sie heute schon kennen, die Akkreditierung sowie die Anerkennung ausländischer Diplome. Darüber stülpt sich das Akkreditierungsdispositiv, bestehend aus einem Organ mit Expertenkommissionen und einer Entscheidungsinstanz. Am Ende der Kette finden sich das Bundesamt für Gesundheit (BAG) und der Chef des Departements des Innern, bei denen die Schlussentscheide liegen. Die FMH wird in diesem Kontext zu einem Ausführungsorgan, das sich um die Weiterbildung der Ärzte und um die Titelerteilung kümmert.

$\mathrm{Zu}$ Beginn fusste das Gesetzesmodell auf vorwiegend hierarchischen Beziehungen unter Partnern. Unter diskreter Mithilfe von Prof. Zeltner und mit viel Anstrengung ist es uns gelungen, es mehr in eine Richtung zu lenken, wo die Beziehung zwischen Medizinalberufekommission und BAG auf einem Dialog basiert. Ausserdem behält die FMH weitgehend ihre Vorrechte, denn landesweit kann nur eine einzige Organisation in der Regel eine gesamtschweizerische, die zudem über die entsprechende Infrastruktur verfügt - das Mandat für die Durchführung der Weiterbildung zugesprochen erhalten.

Der andere Gesetzesteil, der die Bewilligung zur Berufsausübung betrifft, erteilt jetzt die Kompetenz an die Kantone, technische oder räum- liche Kriterien zu formulieren, die eine Planung der ärztlichen Demographie ermöglichen sollen. Mit andern Worten: eine Softversion, aber wesentlich intelligenter als der Zulassungsstopp. Überdies wird die ärztliche Berufsausübung mit Berufspflichten wie Fortbildung, Wahrung der Grenzen der erworbenen Kompetenzen, Berufsgeheimnis und Lauterkeit in der Werbung verbunden. Bei Verletzung dieser Berufspflichten drohen Sanktionen, die von Verwarnung über Bussen bis hin zum befristeten oder definitiven Berufsausübungsverbot reichen. Alle Bemühungen, diese vom Gesetz her zwingenden Bestimmungen durch Anreize und positive Formulierungen zu ersetzen oder sie in unsere Standesordnung zu integrieren - ohne selbstverständlich dem Bundesrat die Möglichkeit zur «Regelung von Einzelheiten» zu nehmen -, all diese Bemühungen sind gescheitert.

Während der ganzen Debatte waren die Gefühle der Kommissionsmitglieder ausserordentlich gespalten. Während die einen die FMH als manipulierende und durchtriebene Lobbyistin wahrnahmen, die sie unter Wahrung eigennütziger Interessen zu überlisten suchte, sahen die anderen in ihr einen zwar achtenswerten, seiner wichtigen Stellung wegen potentiell aber umso gefährlicheren Partner. Und dann tauchten natürlich auch die altbekannten abstrusen Geschichten auf. Es liegt noch eine Menge Arbeit vor uns, bis wir dieses tiefsitzende Misstrauen uns gegenüber losgeworden sind. Nur wenn wir unsere berufliche Befriedigung mit unserer gesundheitspolitischen Verantwortung vereinen, können wir zu einem vertrauenswürdigen Partner werden. Dies aber ist gleichsam unabdingbare Voraussetzung, um legitime Ansprüche qualitativer und materieller Art geltend machen zu können. Oder passt dieses Ideal etwa nicht mehr in eine Konsumgesellschaft, die keine Ideale mehr hat? Auf die Überzeugung kommt es an - muss es ankommen!

Dr. Yves Guisan, Nationalrat, Vizepräsident FMH 


\section{LPméd: lobbying ou partenaire de confiance?}

La Commission de sécurité sociale et de santé du Conseil national a débattu les 27 et 28 avril dernier de la nouvelle Loi sur les professions médicales (LPméd), qui ne traite pas seulement de la formation, mais aussi de l'autorisation de pratiquer. Le projet relève d'une mécanique relativement compliquée avec mise en place non seulement d'une Commission des professions médicales (CPM) regroupant les représentants de la Confédération, des cantons, des associations professionnelles - la loi concerne aussi les dentistes, les pharmaciens et les vétérinaires - et les universités. En cela elle représente le noyau central de la négociation entre partenaires avec des compétences multiples comprenant entre autres la surveillance des examens fédéraux telle que nous la connaissons déjà, la stratégie d'accréditation, ainsi que la reconnaissance des diplômes étrangers. Là-dessus se greffe le dispositif d'accréditation constitué par une organisation avec des commissions d'experts et une instance de décision. Au bout de la chaîne se trouve l'Office fédéral de la santé publique (OFSP) et le chef du Département fédéral de l'intérieur qui prennent la décision finale. Dans ce contexte la FMH devient une instance d'exécution avec pour mission de gérer la formation postgrade des médecins et d'octroyer les titres.

Le modèle était conçu initialement en fonction de rapports essentiellement hiérarchiques entre partenaires. Avec la complicité discrète du Prof. Zeltner mais non sans peine, nous sommes parvenus à le faire évoluer en direction d'une relation de dialogue entre la CPM et l'OFSP. Par ailleurs la FMH garde largement ses prérogatives, puisque pour le pays, c'est une seule organisation, en principe de dimension nationale, et encore pour autant qu'elle dispose d'une infrastructure complète à cet effet, qui peut recevoir le mandat de la prise en charge d'une filière de formation postgrade.

L'autre volet de la loi, qui concerne l'autorisation de pratiquer, a désormais donné la com- pétence aux cantons de l'assortir de conditions techniques ou géographiques afin de permettre une planification de la démographie médicale, en d'autres termes une version soft mais plus intelligente de la clause du besoin. De plus la pratique médicale est assortie de devoirs professionnels, formation continue, respect des limites des compétences acquises, secret professionnel, et honnêteté de la publicité. Ces exigences sont assorties de sanctions allant du blâme jusqu'à la suspension temporaire ou définitive de l'autorisation de pratiquer en passant par l'amende. Dans ce contexte toute tentative de formuler ces dispositions à caractère contraignant de manière plutôt incitatrice et positive, ou bien d'incorporer notre code de déontologie tout en donnant au Conseil fédéral la possibilité de «régler les détails» se sont heurtées à un échec.

Tout au long des débats les sentiments des commissaires sont apparus extrêmement partagés entre la perception d'une FMH lobbyiste, manipulatrice et combinarde, susceptible de tromper leur vigilance au profit d'intérêts manifestement inavouables, et celle d'un partenaire à la rigueur respectable, mais néanmoins potentiellement d'autant plus dangereux que son rôle est plus important. Et chaque fois des anecdotes de boulevard dévastatrices ont inévitablement refait surface. Il y a donc du travail sur la planche pour se débarrasser de cette image de profonde méfiance qui nous colle à la peau. Ce n'est qu'en associant notre satisfaction professionnelle à nos responsabilités de santé publique que nous obtiendrons la qualité de partenaire de confiance, condition sine qua non aussi pour faire valoir des exigences légitimes sur le plan qualitatif et matériel. Idéal qui fait peut-être tache dans une société de consommation qui n' en a plus? C'est surtout une conviction qui s'impose et doit s'imposer!

Dr Yves Guisan, Conseiller national, Vice-président FMH 